Case Report

\title{
First Case Report of Transvenous Pacemaker Placement in a Patient with Congenital Absence of the Clavicles Due to Cleidocranial Dysplasia
}

\author{
Krista Diane Niezwaag ${ }^{1}$, Benjamin James Kotur ${ }^{2}$, Andrew David Michaels ${ }^{1,2, *}$ \\ ${ }^{1}$ Department of Cardiology, Idaho College of Osteopathic Medicine, Meridian, Idaho, the United States \\ ${ }^{2}$ Department of Cardiology, Saint Alphonsus Medical Center, Nampa, Idaho, the United States
}

Email address:

andrew.michaels@saintalphonsus.org (A. D. Michaels)

${ }^{*}$ Corresponding author

To cite this article:

Krista Diane Niezwaag, Benjamin James Kotur, Andrew David Michaels. First Case Report of Transvenous Pacemaker Placement in a Patient with Congenital Absence of the Clavicles Due to Cleidocranial Dysplasia. Cardiology and Cardiovascular Research.

Vol. 5, No. 2, 2021, pp. 94-96. doi: 10.11648/j.ccr.20210502.17

Received: May 12, 2021; Accepted: June 2, 2021; Published: June 15, 2021

\begin{abstract}
Cleidocranial dysplasia is a rare, autosomal dominant disease that is associated with clavicular absence or hypoplasia. Permanent pacemakers are most commonly implanted using percutaneous subclavian venous access. The clavicle is typically used as a bony landmark to guide venous access. Transvenous pacemaker implantation in the setting of clavicular hypoplasia, resection or other anomalies has not been described in literature. This is the first case report of a patient with clavicular absence undergoing transvenous permanent pacemaker implantation. This patient has a rare condition called cleidocranial dysplasia resulting in the congenital absence of his clavicles, along with other skeletal abnormalities. Cardiac anomalies are not associated with this disorder. This patient presented for permanent pacemaker placement in the setting of trifascicular block, symptomatic intermittent second-degree Mobitz type II atrioventricular block, and syncope. Using intra-procedural subclavian venography and intraprocedural Sonosite ultrasound imaging to identify vascular anatomy and surrounding anatomic landmarks, this patient underwent successful placement of a dual chamber transvenous pacemaker. Images from the intraprocedural venogram and the post-procedure chest x-ray illustrate the anatomy in this patient with congenital absence of the clavicles. This case has important implications in subclavian access and pacemaker placement in patients with clavicular abnormalities that may include absent, deformed, or resected clavicles.
\end{abstract}

Keywords: Pacemaker, Cleidocranial Dysplasia, Clavicle

\section{Introduction}

Permanent transvenous pacemakers are typically implanted using percutaneous subclavian venous access. The clavicle is used as a bony landmark to guide venous access. Transvenous pacemaker placement in a patient with absent clavicles is a rare event that has not been reported in literature previously. This is the first report of permanent transvenous pacemaker placement in a patient with absent clavicles. The patient has a rare autosomal dominant disorder called cleidocranial dysplasia, which results in associated bilateral clavicular agenesis. Without the traditional skeletal landmarks on the clavicle, successful placement of this pacemaker relied on both intra-procedural venography and ultrasound imaging. This case has important implications beyond this rare disease, including patients with prior clavicular injury, surgical resection, or hypoplasia.

\section{Case Description}

The patient is a 73 year-old gentleman (167 cm height, 85 $\mathrm{kg}$ weight) with a history of systemic hypertension and cleidocranial dysplasia, with related congenital growth deformities of his cranium involving an open fontanelle, dental anomalies with supernumerary teeth, and absent 
clavicles bilaterally. His hypertension was managed with hydrochlorothiazide and losartan. He presented with syncope. His 12-lead electrocardiogram demonstrated normal sinus rhythm with trifascicular block. An ambulatory electrocardiographic monitor demonstrated multiple episodes of symptomatic second-degree Mobitz type II atrioventricular block. Echocardiography showed a normal left ventricular ejection fraction, moderate concentric left ventricular hypertrophy, and no valvular abnormalities.

He underwent elective dual-chamber pacemaker implantation. Intra-procedural left subclavian venography showed an open subclavian vein with absent clavicles (Figure 1). SonoSite intra-procedural ultrasound imaging showed the subclavian vein $3 \mathrm{~cm}$ deep. A standard pacemaker pocket was made. Access into the left subclavian vein was made on the first attempt without difficulty, using the imaging from both the venogram and ultrasound to guide access. The dual-chamber permanent pacemaker was implanted without complication. The post-procedure chest x-ray demonstrates proper device position, again with absent clavicles (Figure 2). $\mathrm{He}$ has had no further episodes of syncope following pacemaker implantation, with follow-up available at two months.

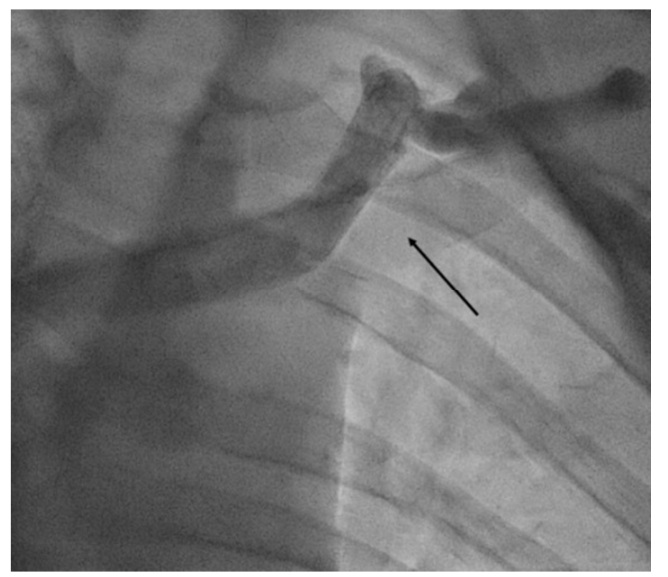

Figure 1. Intraprocedural venogram demonstrating an open left subclavian vein with an absent left clavicle. The first rib is identified by the arrow.

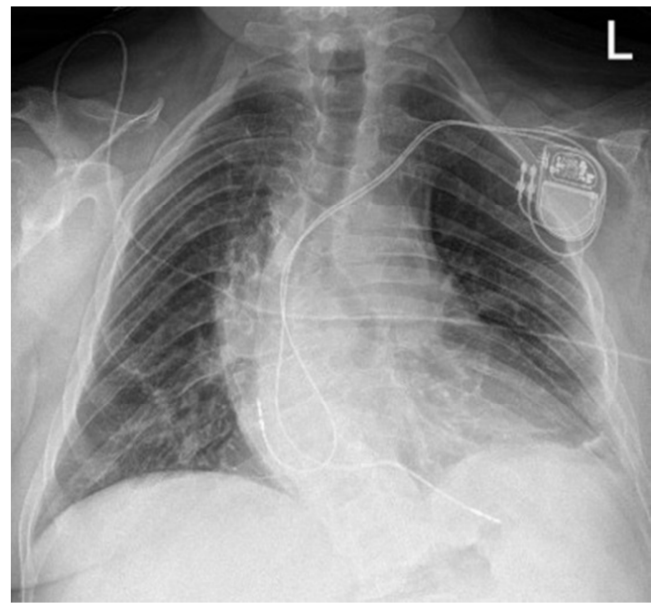

Figure 2. Post-procedure chest x-ray demonstrating proper pacer position, with absent bilateral clavicles.

\section{Discussion}

Cleidocranial dysplasia is a rare, autosomal dominant disorder due to an abnormality in the RUNX2 gene affecting approximately one in one million people [1]. Forty percent of cases occur spontaneously with no familial genetic defect [2]. Patients present with skeletal and dental abnormalities that may include open fontanelles, clavicular hypoplasia, multiple supernumerary teeth, frontal bossing, high palate and short stature [3-6]. The most common presentation of cleidocranial dysplasia is hypoplasia of the clavicular bone, with $10 \%$ of affected individuals presenting with complete absence of the clavicle [7]. Previous case reports have discussed an extreme hypermobility of the shoulder joints, allowing for some patients to approximate their shoulders anteriorly [7]. This disorder has no reported cardiac involvement.

Dual chamber permanent pacemaker placement has a class I indication for symptomatic second-degree Mobitz type II atrioventricular block [8]. In addition, this patient had trifascicular block and syncope. Accessing the subclavian vein traditionally utilizes the bend in the clavicle as a major anatomical landmark for subclavian venous access. However, in a patient with clavicular absence or hypoplasia, use of this landmark is not possible. Without this anatomic landmark for subclavian venous access, other radiological and diagnostic techniques can be used. We described the use of both intraprocedural venography and ultrasound to guide successful venous access. Alternative approaches could include puncturing the axillary vein [9-10], performing a cephalic venous cutdown [11], or implanting a leadless right ventricular pacemaker [12-13].

Subclavian venography has been indicated for, and more commonly used. as a preoperative screening tool for patients with previous transvenous cardiac implantable devices to assess venous lesions and assist in procedural planning [14]. Venography is a safe and effective method for mapping the vascular anatomy, ruling out venous abnormalities, and identifying surrounding anatomic landmarks in patients with clavicular abnormalities.

Vascular ultrasound has been used for subclavian access for several years. A systematic review by Brass and colleagues compared ultrasound guidance versus anatomical landmarks for subclavian and femoral vein catheterization [15]. The authors concluded that ultrasound offered some safety advantages but did not control for user experience or Doppler. In patients with a complete absence of or significant abnormalities in anatomical landmarks, we feel the use of ultrasound-guided access is a safe and effective method for achieving vascular access.

Pacemaker placement in a patient with cleidocranial dysplasia and clavicular absence is a rare event that has not been previously reported. However, this case presents ramifications for techniques applicable in other settings. Loss of normal clavicular anatomy is present in several other rare conditions, including Yunis-Varon Syndrome, pyknodysostosis, mandibuloacral dysplasia, and pseudoarthrosis [16]. Clavicular anomalies can also present 
after injury to the acromioclavicular joint or a conservatively treated, displaced clavicular fracture. We are not aware of other publications discussing permanent transvenous pacemaker implantation in patients with any of these clavicular disorders. Use of intra-procedural venography along with intraprocedural vascular ultrasound offers a safe and effective method for accessing the subclavian vein in these conditions.

In conclusion, this case highlights techniques that can be used for successful permanent transvenous pacemaker implantation in patients with absent clavicles. The use of venography and intraprocedural ultrasound imaging can be employed to help access subclavian venous access in patients without typical clavicular landmarks. This case has implications for pacemaker implantation or subclavian central line placement in patients with absent, deformed, or resected clavicles.

\section{References}

[1] Cleidocranial dysplasia. Genetic and Rare Diseases Information Center (GARD) - an NCATS Program. Accessed March 28, 2021. https://rarediseases.info.nih.gov/diseases/6118/cleidocranial-d ysplasia.

[2] Paul SA, Simon SS, Karthik AK, Chacko RK, Savitha S. (2015) A review of clinical and radiological features of cleidocranial dysplasia with a report of two cases and a dental treatment protocol. Journal of Pharmacy \& Bioallied Sciences, 7 (6), 428-432.

[3] Farrow E, Nicot R, Wiss A, Laborde A, Ferri J. (2018) Cleidocranial dysplasia: a review of clinical, radiological, genetic implications and a guidelines proposal. Journal of Cardiofacial Surgery, 29 (2), 382-389.

[4] Kutilek S, Machytka R, Munzar P. (2019) Cleidocranial dysplasia. Sudanese Journal of Paediatrics, 19 (2), 165-168.

[5] Mundlos S. (1999) Cleidocranial dysplasia: clinical and molecular genetics. Journal of Medical Genetics, 36 (3), 177-182.

[6] Azevedo Almeida LC, Faraj de Lima FB, Matushita H, Valenca MM, Ferreira Castro TL, de Mendonca RN. (2020) Cleidocranial dysplasia, a rare skeletal disorder with failure of the cranial closure: case-based update. Child's Nervous System, 36, 2913-2918.
[7] Dhiman NK, Singh AK, Sharma NK, Jaiswara C. (2014) Cleidocranial dysplasia. National Journal of Maxillofacial Surgery, 5 (2), 206-208.

[8] Kusumoto FM, Schoenfeld MH, Barrett C, Edgerton JR, Ellenbogen KA, Gold MR, Goldschlager NF, Hamilton RM, Joglar JA, Kim RJ, Lee R, Marine JE, McLeod CJ, Oken KR, Patton KK, Pellegrini CN, Selzman KA, Thompson A, Varosy PD. (2019) 2018 ACC/AHA/HRS guideline on the evaluation and management of patients with bradycardia and cardiac conduction delay: a report of the American College of Cardiology/American Heart Association task force on clinical practice guidelines and the Heart Rhythm Society. Circulation, 140 (8), e382-e482.

[9] Migliore F, Curnis A, Bertaglia E. (2016) Axillary vein technique for pacemaker and implantable defibrillator leads implantation: a safe and alternative approach? Journal of Cardiovascular Medicine, 17 (4), 309-313.

[10] Sharma G, Boopathy Senguttuvan N, Thachil A, Leong D, Naik N, Yadav R, Juneja R, Bahl VK. (2012) A comparison of lead placement through the subclavian vein technique with fluoroscopy-guided axillary vein technique for permanent pacemaker insertion. Canadian Journal of Cardiology, 28 (5), $542-546$.

[11] Parsonnet V, Roelke M. (1999) The cephalic vein cutdown versus subclavian puncture for pacemaker/ICD lead implantation. Pacing and Clinical Electrophysiology, 22 (5), 695-697.

[12] Tjong FVY, Reddy VY. (2017) Permanent leadless cardiac pacemaker therapy: a comprehensive review. Circulation, 135 (15), 1458-1470.

[13] Della Rocca DG, Gianni C, Di Biase L, Natale A, Al-Ahmad A. (2018) Leadless pacemakers: state of the art and future perspectives. Cardiac Electrophysiology Clinics, 10 (1), 17-29.

[14] Albertini CM de Moraes, da Silva KR, Leal Filho JM da Motta, Crevelari ES, Filho MM, Carnevale FC, Costa R. (2018) Usefulness of preoperative venography in patients with cardiac implantable electronic devices submitted to lead replacement or device upgrade procedures. Arquivos Brasileiros de Cardiologia 111 (5), 686-696.

[15] Brass P, Hellmich M, Kolodziej L, Schick G, Smith AF. (2015) Ultrasound guidance versus anatomical landmarks for subclavian or femoral vein catheterization. Cochrane Database of Systematic Reviews, 1 (1), CD011447.

[16] Lotlikar PP, Creanga AG, Singer SR. (2018) Clinical and radiological findings in a severe case of cleidocranial dysplasia. BMJ Case Reports, bcr2018226671. 